\title{
Limitations of Tc99m-MIBI-SPECT Imaging Scans in Persistent Primary Hyperparathyroidism
}

\author{
Janneke E. Witteveen · Job Kievit • \\ Marcel P. M. Stokkel · Hans Morreau • \\ Johannes A. Romijn • Neveen A. T. Hamdy
}

Published online: 19 October 2010

(C) The Author(s) 2010. This article is published with open access at Springerlink.com

\begin{abstract}
Background In primary hyperparathyroidism (PHPT) the predictive value of technetium $99 \mathrm{~m}$ sestamibi single emission computed tomography (Tc99m-MIBI-SPECT) for localizing pathological parathyroid glands before a first parathyroidectomy (PTx) is $83-100 \%$. Data are scarce in patients undergoing reoperative parathyroidectomy for persistent hyperparathyroidism. The aim of the present study was to determine the value of Tc99m-MIBI-SPECT in localizing residual hyperactive parathyroid tissue in patients with persistent primary hyperparathyroidism (PHPT) after initial excision of one or more pathological glands.

Method We retrospectively evaluated the localizing accuracy of Tc99m-MIBI-SPECT scans in 19 consecutive patients with persistent PHPT who had a scan before reoperative parathyroidectomy. We used as controls 23 patients with sporadic PHPT who had a scan before initial surgery.
\end{abstract}

J. E. Witteveen · J. A. Romijn · N. A. T. Hamdy $(\bowtie)$

Department of Endocrinology \& Metabolic Diseases, Leiden University Medical Center, Albinusdreef 2, 2333 ZA Leiden, The Netherlands

e-mail: N.A.T.Hamdy@LUMC.nl

J. Kievit

Department of Surgery, Leiden University Medical Center, Albinusdreef 2, 2333 ZA Leiden, The Netherlands

M. P. M. Stokkel

Department of Nuclear Medicine, Leiden University Medical Center, Albinusdreef 2, 2333 ZA, Leiden, The Netherlands

H. Morreau

Department of Pathology, Leiden University Medical Center, Albinusdreef 2, 2333 ZA Leiden, The Netherlands
Results In patients with persistent PHPT, Tc99m-MIBISPECT accurately localized a pathological parathyroid gland in $33 \%$ of cases before reoperative parathyroidectomy, compared to $61 \%$ before first PTx for sporadic PHPT. The Tc99m-MIBI-SPECT scan accurately localized intra-thyroidal glands in 2 of 7 cases and a mediastinal gland in 1 of 3 cases either before initial or reoperative parathyroidectomy.

Conclusions Our data suggest that the accuracy of Tc99m-MIBI-SPECT in localizing residual hyperactive glands is significantly lower before reoperative parathyroidectomy for persistent PHPT than before initial surgery for sporadic PHPT. These findings should be taken in consideration in the preoperative workup of patients with persistent primary hyperparathyroidism.

\section{Introduction}

Primary hyperparathyroidism is one of the most common endocrine disorders. Surgical removal of all pathological parathyroid tissue is the only therapy that leads to definitive and durable cure. The need for preoperative localization of pathological parathyroid gland(s) before initial surgery depends on the chosen surgical approach. In case of traditional bilateral neck exploration, localization studies are not deemed necessary because experienced surgeons have, on average, a 98\% chance of excising all pathological tissue, as the procedure entails visualization of all four parathyroid glands [1, 2]. However, localization studies become mandatory when the surgeon opts for a more focused unilateral or minimally invasive approach. Accurate localization studies are even more important before reoperative parathyroidectomy for persistent hyperparathyroidism, as a second (or more) neck exploration is 
technically more challenging than initial surgery and may be associated with as much as a threefold increase in morbidity [1, 3-5]. Persistent hyperparathyroidism is reported to occur in $2-7 \%$ of patients who have had a parathyroidectomy $[1,6,7]$. Excluding insufficient experience of the operating surgeon, persistent PHPT may be caused by a second adenoma, multiple gland hyperplasia, an ectopically located hyperactive gland undetected at initial surgery, or, rarely, parathyromatosis from gland spillage during initial surgery [8].

Technetium 99m sestamibi (Tc99m-MIBI), particularly when complemented by single emission computed tomography (Tc99m-MIBI-SPECT), is a widely used imaging technique for the preoperative localization of parathyroid adenomas [1, 9]. Tc99m-MIBI-SPECT has a sensitivity ranging from 66 to $90 \%$ and a positive predictive value ranging from 83 to $100 \%$ for pathological parathyroid glands [1, 10-17]. However, the test is also known to have lower sensitivity for small adenomas weighing $<500 \mathrm{mg}[15,18]$ and in the presence of multiple gland pathology $[1,9,15,17-20]$.

Tc99m-MIBI was originally developed for myocardial perfusion imaging. It is a monovalent lipophilic cation that diffuses passively through cell membranes and accumulates almost exclusively in mitochondria following negative membrane potentials [21]. The imaging technique has been used to detect benign tumors as well as several primary malignancies and metastatic tumors [22]. In primary hyperparathyroidism, the mechanism by which Tc99mMIBI can localize a pathological parathyroid gland is based on the increased uptake of the radiopharmaceutical by functionally hyperactive glands; on the abundance of mitochondria in parathyroid oxyphil cells, which sequester sestamibi intracellularly; and on the increased perfusion of hyperactive parathyroid glands. However, imaging studies are often negative in persistent hyperparathyroidism. Potential contributory factors to false negative scans are a postoperative disturbance in the perfusion of remaining pathological glands and a predominance of parathyroid chief cells, which are poorer in mitochondrial content compared to oxyphil cells [18].

In patients undergoing reoperative parathyroidectomy for persistent hyperparathyroidism, the accuracy of Tc99mMIBI-SPECT in localizing hyperactive parathyroid tissue has been largely assessed in mixed patient groups, including patients in whom no parathyroid tissue was found at initial surgery [23, 24], patients with a known MEN-1 (multiple endocrine neoplasia type 1) mutation [25], and patients with secondary or tertiary hyperparathyroidism $[26,27]$. Data on the ability of Tc99m-MIBI-SPECT to detect and localize residual hyperactive parathyroid tissue in patients with persistent primary hyperparathyroidism are particularly scarce. The available data do suggest, however, that the predictive value of this technique falls dramatically from $80 \%$ or higher before initial surgery for single gland disease (SGD) to as low as $50 \%$ before reoperative parathyroidectomy for persistent PHPT [28-30]. Because reoperative parathyroidectomy may be associated with increased morbidity, accurate localization of residual hyperactive tissue becomes of paramount importance in patients with persistent PHPT [28-30]. Correct preoperative localization of a hyperactive parathyroid gland by Tc99m-MIBI-SPECT would allow for more focused and efficient exploration, thus reducing the risk of damage to the recurrent laryngeal nerve(s) and significantly increasing the likelihood of cure.

The aim of the present study was to determine the ability of Tc99m-MIBI-SPECT to detect and localize residual hyperactive parathyroid glands prior to reoperative parathyroidectomy in patients with persistent PHPT.

\section{Patients}

Using hospital records, we identified 19 consecutive patients who required reoperative parathyroidectomy for persistent hyperparathyroidism and who had had parathyroid localization studies with Tc99m-MIBI-SPECT before surgery. These were 14 women and 5 men with a mean age of $55 \pm 12$ years at diagnosis, who had undergone between one and three unsuccessful earlier neck explorations. Sixteen of the 19 patients had their initial surgery and earlier reoperations, if applicable, at other institutions and were referred to our unit for further workup before reoperative parathyroidectomy. We compared these patients with 23 patients with sporadic primary hyperparathyroidism due to single gland disease who also had had a Tc99mMIBI-SPECT scan prior to initial surgery. These were, respectively, 21 women and 2 men with a mean age of $59 \pm 12$ years at diagnosis. All surgical procedures were undertaken by two surgeons with considerable experience in endocrine surgery.

\section{Methods}

Demographic data, operative data, preoperative and postoperative laboratory data, and histological findings were obtained from patients' hospital records. In the present study, cure is defined as sustained normal serum calcium and PTH concentrations more than 6 months after parathyroidectomy [6]. Persistent hyperparathyroidism is defined as persistently elevated serum calcium and PTH concentrations in consecutive samples within and beyond 6 months after surgery [6]. 
Histological preparations were independently reviewed by an experienced pathologist. A diagnosis of parathyroid adenoma was based on the presence of a benign encapsulated neoplasm usually involving a single gland with an adjacent rim of normal glandular tissue [31]. Parathyroid hyperplasia was defined as an absolute increase in parathyroid parenchymal cell mass resulting from proliferation of chief cells, oncocytic cells, and transitional oncocytic cells in multiple parathyroid glands in the absence of a known stimulus for PTH hypersecretion such as renal failure or vitamin D deficiency [31]. A diagnosis of hyperplasia was also favored when synchronous multiglandular disease was found at operation.

\section{Tc99m-MIBI-SPECT imaging technique}

The Tc99m-MIBI-SPECT scan was performed as follows: after intravenous injection of $500 \mathrm{MBq}$ of Tc99m methoxy-isobutyl-isonitrile (MIBI), planar images of the head and neck region and chest were performed at a matrix size of $256 \times 256$ (10 min per frame). Scintigraphy was performed as a dual-phase single tracer examination. Images were acquired with the patient in the supine position, $15 \mathrm{~min}$ and $2 \mathrm{~h}$ after injection of the radiopharmaceutical. A gamma camera (Toshiba GCA-7200, Tokyo, Japan) equipped with low-energy high-resolution (LEHR) collimators was used for image acquisition. Single photon emission computed tomography (SPECT) was performed in a $128 \times 128$ matrix size, using a step angle of $4^{\circ}$ and a step time of $35 \mathrm{~s}$ per step, $90 \mathrm{~min}$ after the injection. The filtered back projection was used for image reconstruction, using a Butterworth filter (8 order, subset 12). All Tc99mMIBI-SPECT scans were reviewed by an experienced nuclear medicine physician who was blinded to the outcome of the surgical procedure.
Surgical procedures

Bilateral neck exploration consisted of visualization of all four parathyroid glands, followed by either excision of a single enlarged parathyroid gland or subtotal or total parathyroidectomy with auto transplantation if more than one parathyroid gland was found to be enlarged. If not all four parathyroid glands could be visualized and no enlarged parathyroid gland was found, dissection of the ipsilateral anterior compartment extending from the level of the hyoid bone superiorly to the suprasternal notch inferiorly and hemithyroidectomy were undertaken on the side of the missing parathyroid. Unilateral neck exploration and minimally invasive neck exploration were guided by intraoperative PTH measurement.

\section{Analysis of data}

The ability of Tc99m-MIBI-SPECT to detect and localize hyperactive parathyroid tissue was evaluated in three different ways, thereby providing the answers to three different questions that are clinically relevant to the operating surgeon.

The first question we addressed is "Is the Tc99m-MIBISPECT scan able to detect any parathyroid hyperactivity?" This question relates to the overall sensitivity of the scan for detecting the presence of hyperactive parathyroid tissue in the scanned neck and mediastinal regions (Fig. 1). A scan was considered true positive when it correctly identified the presence of an abnormal gland at any site. A scan was considered true negative when no pathological parathyroid gland(s) was found at surgery and no presence of hyperactive parathyroid tissue was suggested by the scan. A scan was considered false positive when no pathological glands were found at operation, although the scan had suggested the presence of hyperactive parathyroid tissue.
Fig. 1 Positive Tc99m-MIBISPECT imaging scan demonstrating increased radioactive pharmaceutical uptake in the right lower neck suggesting a pathological parathyroid gland in this location (a), concurring with subsequent operative findings of a $2.5 \mathrm{~cm}$ pathological parathyroid adenoma (b)
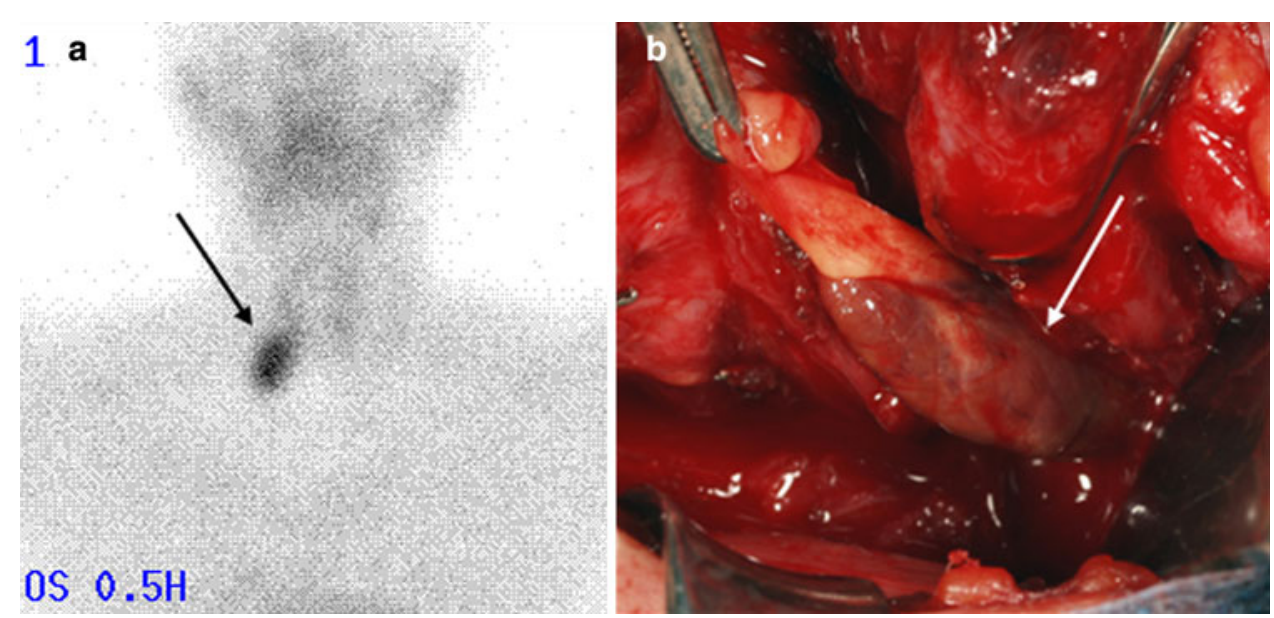
A scan was considered false negative when a pathological parathyroid gland was removed at surgery, although the scan had not demonstrated the presence of hyperactive parathyroid tissue.

The second question addressed is "Is the scan able to lateralize the area of hyperactive parathyroid tissue, thus indicating which side of the neck should be explored?" This question relates to the sensitivity and specificity of the scan in identifying the side of the neck in which hyperactive parathyroid tissue is localized (Fig. 2a). A true positive side was defined as a neck side from which a pathological parathyroid gland was removed at surgery, and in which the presence of hyperactive parathyroid tissue was suggested by the preoperative localization test. A true negative side was defined as a neck side in which no pathological parathyroid gland was found at surgery, and in which no presence of hyperactive parathyroid tissue was suggested by the preoperative localization test. A false positive side was defined as a neck side in which no pathological parathyroid gland was found at surgery, although the presence of hyperactive parathyroid tissue was suggested by the preoperative localization test. A false negative side was defined as a neck side from which a pathological parathyroid gland was removed at surgery, although no presence of hyperactive parathyroid tissue was suggested by the preoperative localization test.

The third question is "Is the scan able to accurately localize the region of the neck where the hyperactive parathyroid tissue is present?", thus providing an even smaller operative field for the surgeon to focus on. This third question takes accuracy of localization of the imaging modality a step further by identifying the localization of the hyperactive parathyroid tissue in any of 5 designated anatomical areas of the neck (four thyroid quadrants, and a fifth "elsewhere" area combining all possible ectopic localizations in the mediastinum) (Fig. 2b). With respect to the risk of damaging the recurrent laryngeal nerve, a correct answer to question 2 would limit the risk to one nerve only, whereas a correct answer to question 3 would provide additional information on the magnitude of this risk by identifying the specific region to be explored. A true positive region was defined as a region from which a pathological parathyroid gland was removed at surgery, and in which the presence of hyperactive parathyroid tissue was suggested by the preoperative localization test. A true negative region was defined as a region in which no pathological parathyroid gland was found at surgery, and in which no presence of hyperactive parathyroid tissue was suggested by the preoperative localization test. A false positive region was defined as a region in which no pathological parathyroid gland was found at surgery, although the presence of hyperactive parathyroid tissue was suggested in this region by the preoperative localization test. A false negative region was defined as a region from which a pathological parathyroid gland was removed at surgery, although no presence of hyperactive parathyroid tissue was suggested in this region by the preoperative localization test.

True negative and false positive scans, sides or regions could only be determined in patients who were cured after the surgery by the finding and removal of a pathological parathyroid gland elsewhere in the neck or mediastinum

Fig. 2 Analysis of the ability of Tc99m-MIBI-SPECT scan to correctly localize a pathological parathyroid gland as confirmed by localization at surgery by dividing the neck into two anatomical areas (the right and left side of the neck) (a) or into five anatomical areas (the four thyroid quadrants and a fifth area of all possible ectopic localizations in the mediastinum) (b)
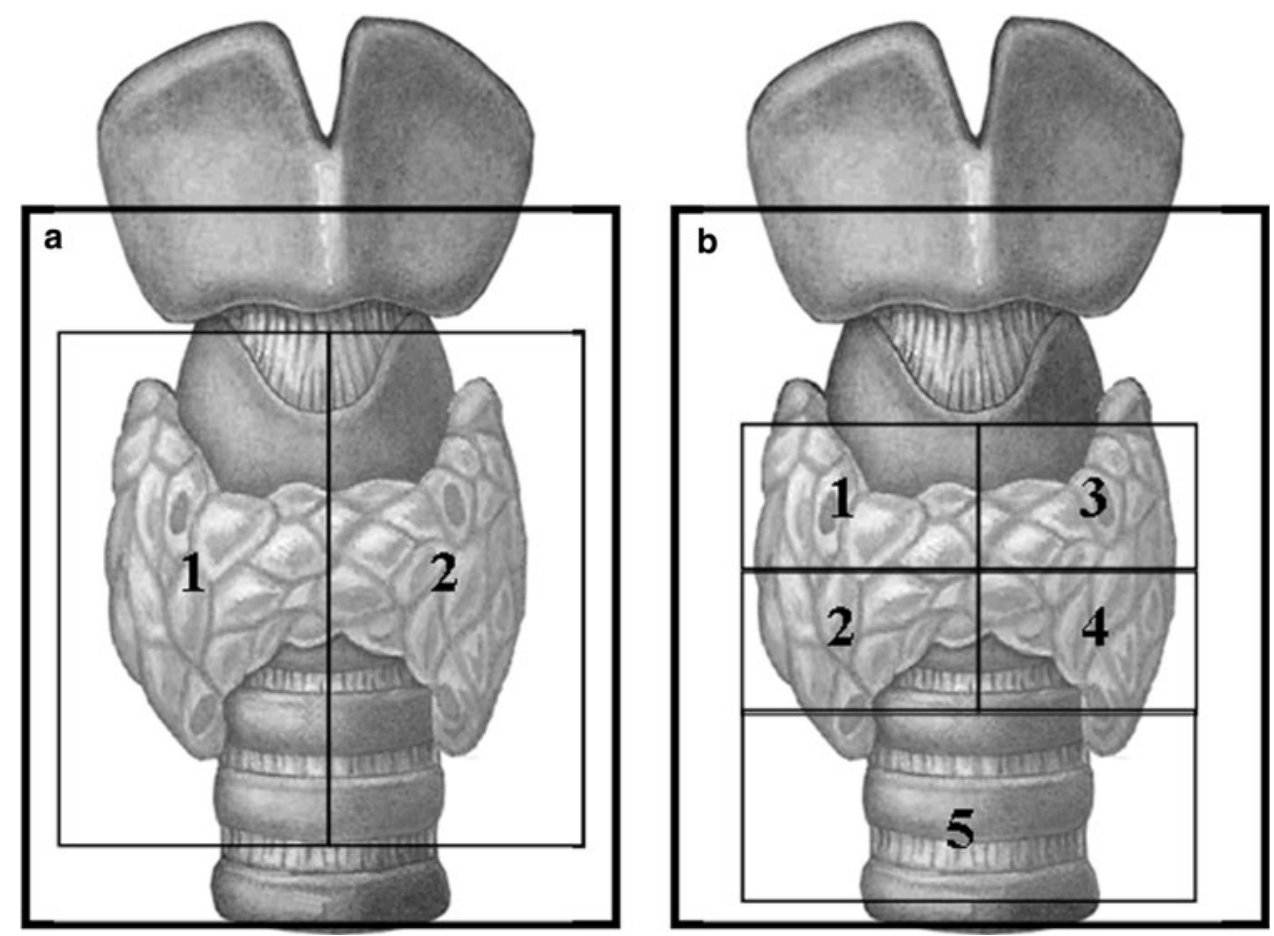
(this latter condition precluding comprising the sensitivity and specificity by false negative surgery).

In all three analyses widely accepted definitions for sensitivity and specificity were used to address each of the three different questions posed. Sensitivity was thus judged to be the power of the test to identify the presence of pathological parathyroid glands at any site regardless of accuracy of localization (question 1), on the correct side of the neck (question 2), or in the exact region of neck (question 3). Specificity was judged to be the power of Tc99m-MIBISPECT to recognize the absence of a pathological parathyroid gland in the truly negative side of the neck (question 2 ) or in the exact region of the neck (question 3 ).

\section{Statistical analysis}

Statistical analysis was performed with SPSS 16 software (SPSS, Inc., Chicago, IL). Results are expressed as mean \pm SD unless otherwise stated. Baseline characteristics of the patient groups were compared with one-way analysis of variance (ANOVA) for continuous variables, and the chi-square statistic was used for comparison of categorical variables. A probability level of random difference of $P<0.05$ was considered significant.

\section{Results}

Demographic data

Patients who underwent reoperative parathyroidectomy for persistent PHPT did not differ significantly in age and
Fig. 3 Flowchart of the answers to the three clinical questions addressed in 19 patients who had preoperative parathyroid localization studies with Tc99m-MIBI-SPECT prior to reoperative parathyroidectomy for persistent PHPT

gender from patients who underwent initial surgery for sporadic PHPT. Also, laboratory data before operation did not differ significantly between the two patient groups (Table 1).

Operative data and postoperative cure

Bilateral neck exploration was more frequently performed in patients with persistent hyperparathyroidism undergoing reoperative parathyroidectomy $(84 \%)$ than in patients with a single adenoma undergoing initial surgery (56\%) (Table 1).

At least one and up to three hyperactive parathyroid glands had been removed at initial surgery in patients with persistent hyperparathyroidism. The 18 pathological parathyroid glands removed at reoperative parathyroidectomy were removed from ectopic locations $(n=8 ; 44 \%)$ : intrathyroidal $(n=4)$, thymus $(n=2)$, mediastinum $(n=$ 1 ), and a location ventral to the trachea $(n=1)$. Other glands were removed from expected anatomical locations in the right lower quadrant $(n=4)$, right upper quadrant $(n=3)$, left lower quadrant $(n=2)$, and left upper quadrant $(n=1)$ of the neck.

Complete cure was eventually achieved in 11 of the 19 patients $(58 \%)$. Of the remaining 8 patients, 6 had no further surgery, 1 had one further operation, and 1 had three further operations, which resulted in improvement in the severity of the hyperparathyroidism but not in complete
Table 1 Demographic, laboratory, operative, and pathology data of patients who had a Tc99m-MIBI-SPECT scan prior to initial surgery for sporadic primary hyperparathyroidism (PHPT) or prior to reoperative parathyroidectomy for persistent PHPT $s$ serum
a Reference range
$2.15-2.55 \mathrm{mmol} / \mathrm{l}$
b Reference range $1.5-8 \mathrm{pmol} / \mathrm{l}$

\begin{tabular}{llll}
\hline & $\begin{array}{l}\text { Persistent PHPT } \\
(n=19)\end{array}$ & $\begin{array}{l}\text { Sporadic PHPT } \\
(n=23)\end{array}$ & $P$ value \\
\hline Age, years $( \pm \mathrm{SD})$ & $55 \pm 12$ & $59 \pm 12$ & 0.274 \\
Sex, M:F & $5: 14$ & $2: 21$ & 0.127 \\
Preoperative laboratory data & & & 0.399 \\
s-Corrected calcium, mmol/l & $2.88 \pm 0.35$ & $2.80 \pm 0.23$ & 0.131 \\
s-PTH, pmol/l & $16.2 \pm 9.3$ & $24.5 \pm 20.2$ & 0.027 \\
Postoperative laboratory data & & & 0.043 \\
s-Corrected calcium, mmol/1 & & \\
s-PTH, pmol/1 & $2.46 \pm 0.28$ & $4.0 \pm 2.7$ & 0.053 \\
Operative data & $10.0 \pm 11.7$ & $13(56 \%)$ & 0.625 \\
Bilateral exploration & & $5(22 \%)$ & 0.030 \\
Unilateral exploration & $16(84 \%)$ & $5(22 \%)$ & 0.037 \\
Minimally invasive & $3(16 \%)$ & & 0.152 \\
Pathology data & 0 & $19(83 \%)$ & 0.210 \\
Adenoma & & $4(17 \%)$ & 0.062 \\
Hyperplasia & $9(42 \%)$ & 0 & $2.03 \pm 1.40$ \\
No pathological glands & $2(11 \%)$ & & \\
Gland diameter, cm $( \pm \mathrm{SD})$ & $1.21 \pm 0.93$ & & \\
\hline
\end{tabular}




\section{Question 1: "Does Tc99m-MIBI-SPECT detect any parathyroid hyperactivity?"}

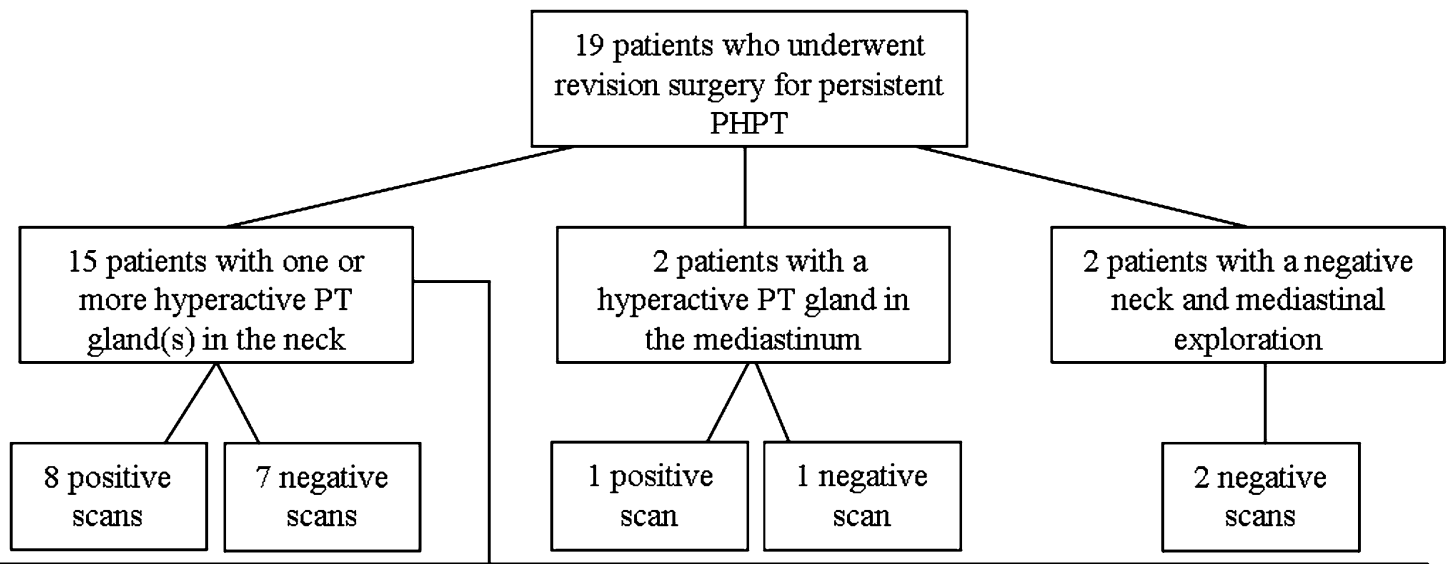

Answer 1: General Sensitivity of the scan is $47 \%(9 / 19)$

Question 2: "Which side of the neck should be explored, left or right?"

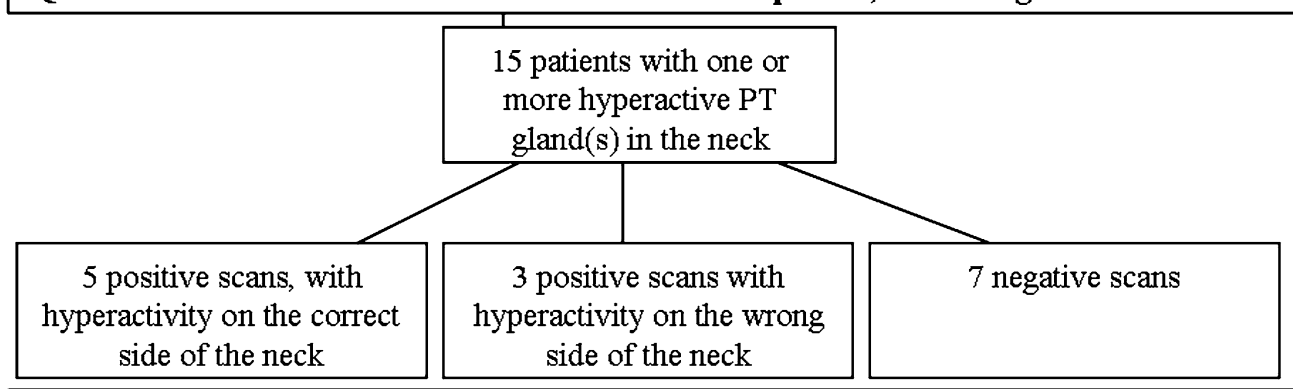

Answer 2: Ability of the scan to localize the gland to the correct side of the neck is $33 \%$ $(\mathbf{5} / \mathbf{1 5})$

Question 3: "Is the scan able to accurately localize a pathological parathyroid gland?"

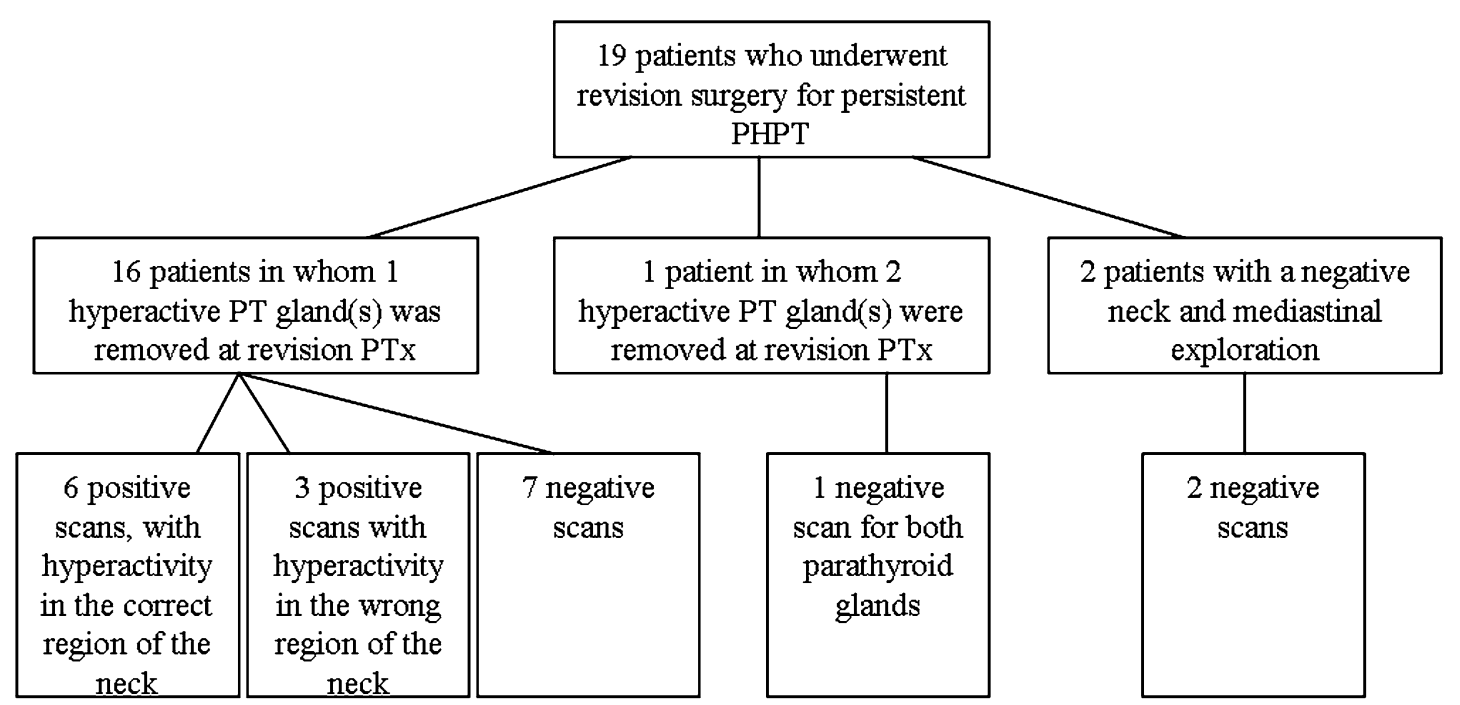

Answer 3: Ability of the scan to predict the exact localization of a hyperactive PT gland is $33 \%(6 / 18)$ 
cure as defined in our study. In all 8 patients the decision to withhold further surgery was based on a combination of lack of severe symptoms, mild biochemical features of hyperparathyroidism, and the increased morbidity risk associated with further surgery in the context of prior extensive neck exploration, which had to be extended to mediastinal exploration in some cases.

The 23 patients with PHPT who had a Tc99m-MIBISPECT scan prior to initial surgery for sporadic PHPT were all cured, and cure was sustained for a mean duration of 47 months (range: 6-188 months) after PTx. Pathological parathyroid glands removed at surgery were predominantly located in the right $(n=10,43 \%)$ or left $(n=7$, $30 \%$ ) lower thyroid quadrants, and only 1 pathological gland was located in the right upper quadrant of the neck. Five successfully removed ectopic glands had been in subcapsular thyroidal $(n=2)$, intrathyroidal $(n=1)$, or mediastinal $(n=2)$ locations.

\section{Histological data}

On histological examination, $42 \%$ of the patients with persistent hyperparathyroidism had a second adenoma and $47 \%$ had hyperplastic parathyroid gland(s) (Table 1). A single adenoma was removed in $83 \%$ of the patients who underwent initial surgery for sporadic PHPT. The average diameter of a pathological parathyroid gland found at reoperative parathyroidectomy for persistent PHPT was smaller $(1.21 \mathrm{~cm})$ compared to the average size of glands found at initial surgery for sporadic PHPT $(2.03 \mathrm{~cm})$.

Value of Tc99m-MIBI-SPECT scans prior to reoperative parathyroidectomy for persistent PHPT

In total, 18 hyperactive parathyroid glands were removed at reoperative parathyroidectomy from 19 patients with persistent PHPT. One hyperactive parathyroid gland was removed in 16 of these patients, 2 glands in 1 patient, and no pathological glands could be found in the other 2
Fig. 4 Flowchart of the answers to the three clinical questions addressed in 23 patients who had preoperative parathyroid localization studies with Tc99m-MIBI-SPECT prior to initial surgery for sporadic PHPT

patients despite extensive bilateral neck and mediastinal explorations. Tc99m-MIBI-SPECT demonstrated increased radiotracer uptake, suggesting parathyroid hyperactivity in only 9 of the 19 patients (47\%) (Fig. 3).

At reoperative parathyroidectomy 15 patients had a pathological gland removed from the neck region. Tc99mMIBI-SPECT was able to identify the correct side of the neck in 5 of these 15 patients (33\%) (Table 2; Fig. 3).

Tc99m-MIBI-SPECT was able to detect and accurately localize only 6 of the 18 pathological glands removed at reoperative parathyroidectomy either from the neck or from ectopic locations (33\%) (Table 3; Fig. 3). Two of these glands, an adenoma and a hyperplastic gland, were found in normal anatomical locations in the left upper and left lower thyroid quadrants, and 4 glands were found in ectopic locations in the neck, one adenoma was in the thymus, one adenoma and one hyperplastic gland were in the thyroid, and one adenoma was located ventral to the trachea, caudally in the neck.

Of the 12 pathological glands not accurately localized by Tc99m-MIBI-SPECT, $67 \%$ were hyperplastic glands and $33 \%$ were adenomas. Only 4 of the 12 glands missed at Tc99m-MIBI-SPECT were found in an ectopic location at surgery: one hyperplastic gland was in the thymus, one adenoma and one hyperplastic gland were in the thyroid gland, and one hyperplastic gland was in the mediastinum. Pathological glands with negative imaging were smaller, with a mean diameter of $9 \mathrm{~mm}$, ranging from 5 to $16 \mathrm{~mm}$, and had a histological predominance of chief cells.

Value of Tc99m-MIBI-SPECT scans prior to initial surgery for sporadic PHPT

All 23 patients with sporadic PHPT had one hyperactive parathyroid gland removed at initial surgery and achieved

Table 2 Predictive value of Tc99m-MIBI-SPECT for the localization of pathological parathyroid glands in the correct side of the neck

\begin{tabular}{|c|c|c|c|c|}
\hline & $\%$ Sensitivity & $\%$ Specificity & $\% \mathrm{PPV}$ & $\% \mathrm{NPV}$ \\
\hline Before initial surgery for sporadic PHPT $(n=23)$ & 67 & 100 & 100 & 75 \\
\hline Before reoperative parathyroidectomy for persistent PHPT $(n=19)$ & 33 & 80 & 63 & 53 \\
\hline
\end{tabular}

$P P V$ positive predictive value, $N P V$ negative predictive value

Table 3 Predictive value of Tc99m-MIBI-SPECT for the localization of pathological parathyroid glands in the exact region of the neck

\begin{tabular}{|c|c|c|c|c|}
\hline & $\%$ Sensitivity & $\%$ Specificity & $\% \mathrm{PPV}$ & $\% \mathrm{NPV}$ \\
\hline Before initial surgery for sporadic PHPT $(n=23)$ & 61 & 99 & 93 & 91 \\
\hline Before reoperative parathyroidectomy for persistent PHPT $(n=19)$ & 33 & 95 & 67 & 84 \\
\hline
\end{tabular}




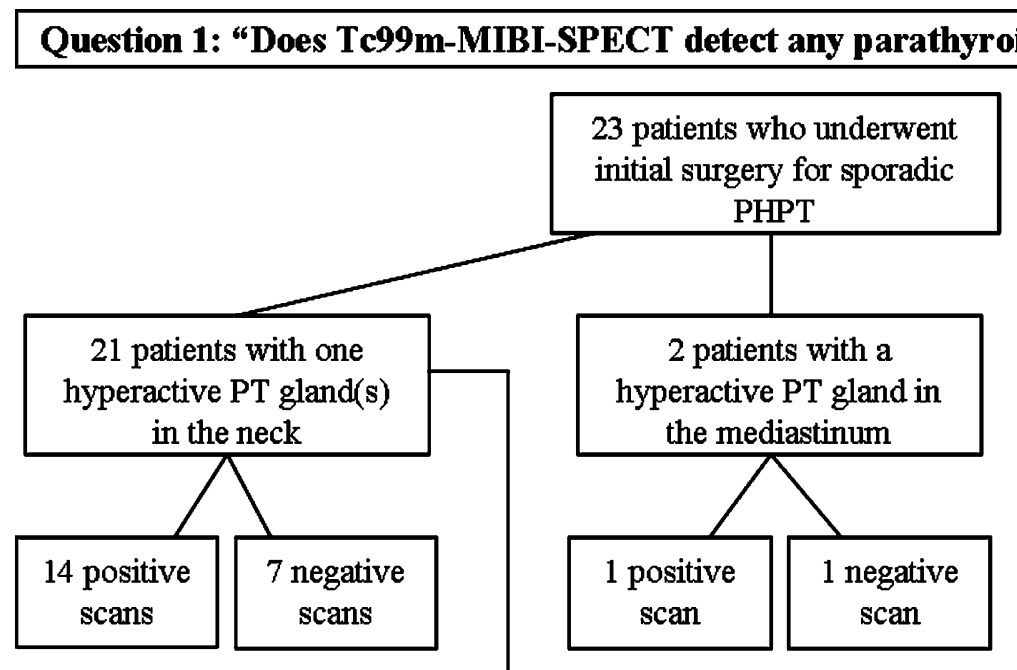

Answer 1: General Sensitivity of the scan is $65 \%(15 / 23)$

Question 2: "Which side of the neck should be explored, left or right?"

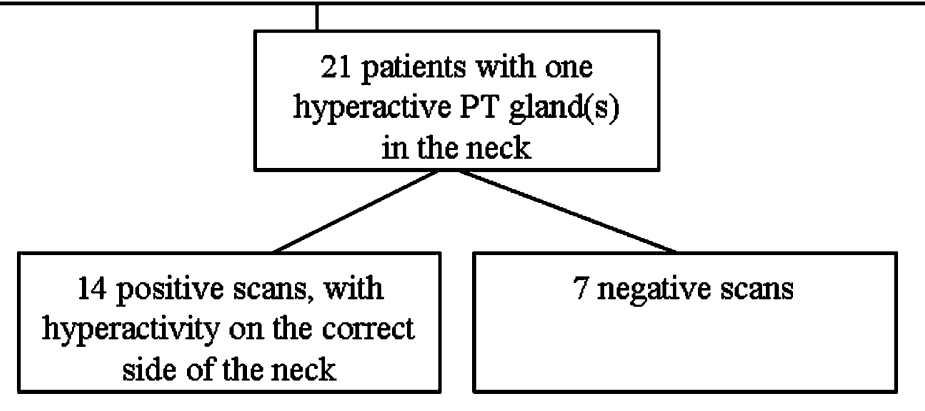

Answer 2: Ability of the scan to localize the gland to the correct side of the neck is $67 \%$ (14/21)

Question 3: "Is the scan able to accurately localize a pathological parathyroid gland?"

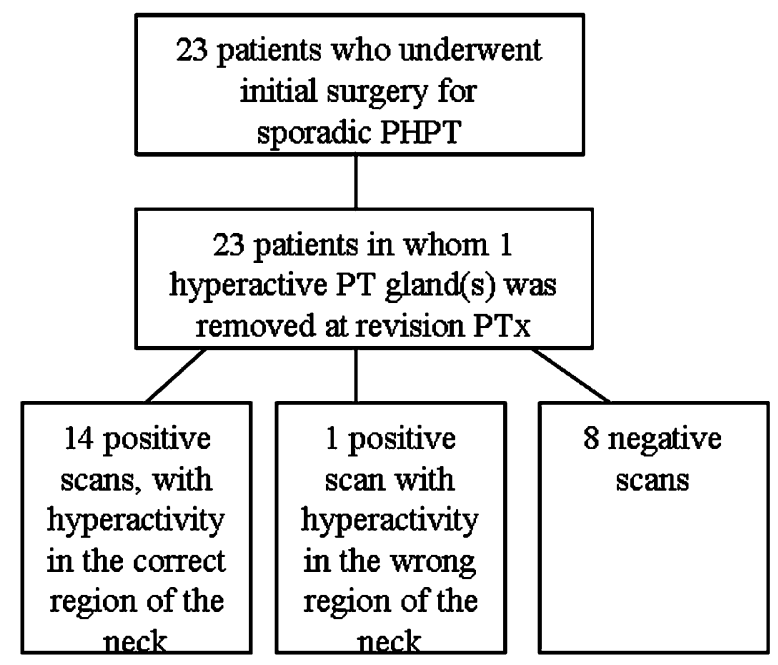

Answer 3: Ability of the scan to predict the exact localization of a hyperactive PT gland is $61 \%(14 / 23)$ 
cure. However, Tc99m-MIBI-SPECT scan demonstrated increased radiotracer uptake, suggesting parathyroid hyperactivity in only 15 of the 23 patients (65\%) (Fig. 4). In 21 of the 23 patients the hyperactive parathyroid gland was removed from the neck. Tc99m-MIBI-SPECT was able to identify the correct side of the neck in 14 of these patients $(67 \%)$ (Table 2; Fig. 4). In 2 of the 23 patients a hyperactive gland was removed from the mediastinum, but Tc99m-MIBI-SPECT was able to detect only one of these glands (Fig. 4).

Tc99m-MIBI-SPECT was able to accurately localize 14 of the 23 pathological parathyroid glands removed at operation (61\%) (Table 3; Fig. 4). Of the 14 glands accurately localized by Tc99m-MIBI-SPECT, 12 (86\%) were adenomas located in the right lower thyroid quadrant in 9 cases $(76 \%)$, in the left lower thyroid quadrant in 2 cases $(17 \%)$, and in the mediastinum in 1 case $(7 \%)$. The remaining 2 accurately localized glands were hyperplastic parathyroid glands located in the left lower thyroid quadrant. Of the 9 pathological glands that were not localized or that were inaccurately localized by Tc99m-MIBI-SPECT, 5 were found in the neck in expected anatomical locations at surgery and 4 were ectopically located: 3 intrathyroidally and 1 in the mediastinum. Intrathyroidal parathyroid glands were found in 2 cases by visualization during extensive bilateral neck exploration and in 1 case during unilateral neck exploration combined with intraoperative PTH (IOPTH) measurement. The mediastinal gland was found during thoracic extension of a full bilateral neck exploration during which no pathological parathyroid glands were found in the neck.

Limitations of Tc99m-MIBI-SPECT imaging studies

\section{Localization ability for pathological parathyroid glands in anatomically expected versus ectopic locations}

Combining all patients included in this study, pathological parathyroid glands were most frequently found in the right (37\%) and left (32\%) lower thyroid quadrants (Fig. 5). Thirteen of the 41 pathological glands removed at surgery (32\%) were found in ectopic locations: intrathyroidally $(n=7)$, in the thymus $(n=2)$, in the mediastinum $(n=3)$, and ventral to the trachea in the neck $(n=1)$. An intrathyroidal parathyroid gland was accurately localized preoperatively in 2 of 7 cases (29\%), a mediastinal gland in 1 of 3 cases (33\%), a gland in the thymus in 1 of 2 cases $(50 \%)$, and a gland ventral to the trachea in one case $(100 \%)$.

Tc99m-MIBI-SPECT scan had a lower sensitivity for pathological parathyroid glands identified at surgery in the upper quadrants of the neck $(2 / 9=22 \%)$, than for glands found in the lower quadrants of the neck $(16 / 28=57 \%)$

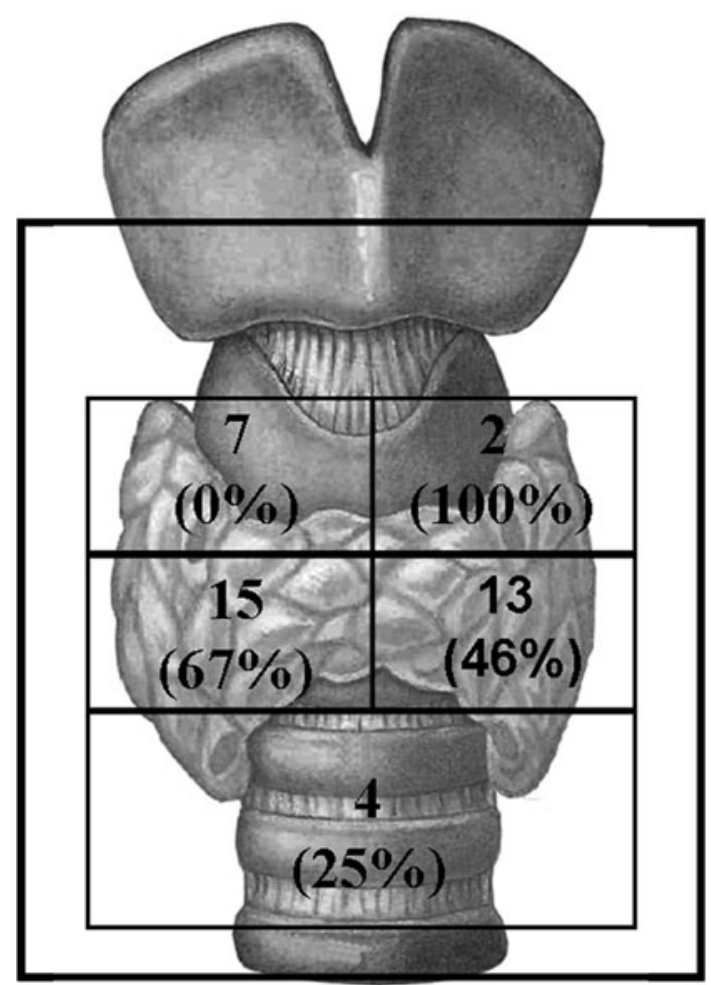

Fig. 5 Combined number of pathological parathyroid glands identified at initial surgery $(n=23)$ and at revision surgery $(n=18)$ in each of the four quadrants of the neck and the mediastinum. The sensitivity of Tc99m-MIBI-SPECT for localization in each of these four quadrants of the neck and the mediastinum is shown in parentheses

(Fig. 5). None of the 7 pathological parathyroid glands in the right upper thyroid quadrant could be accurately localized preoperatively. One of the 7 pathological glands in the right upper thyroid quadrant showed uptake in the right lower quadrant, and the other 6 glands showed no radiotracer uptake on Tc99m-MIBI-SPECT.

\section{Localization ability for adenomas versus hyperplastic pathological glands}

In the present study, Tc99m-MIBI-SPECT scan was able to accurately localize 16 of 26 adenomas (64\%) and 4 of 16 hyperplastic glands (25\%) found at surgery.

\section{Localization ability in relation to pathological parathyroid gland size}

Pathological parathyroid glands with a diameter greater than $1.5 \mathrm{~cm}$ were more frequently accurately localized by Tc99m-MIBI-SPECT than glands with a diameter smaller than $1.5 \mathrm{~cm}$. In patients undergoing initial surgery for sporadic PHPT, Tc99m-MIBI-SPECT had a sensitivity of $71 \%$ for glands with a diameter $>1.5 \mathrm{~cm}$ compared to a 
sensitivity of $44 \%$ for glands with a diameter $<1.5 \mathrm{~cm}$. In patients who underwent reoperative parathyroidectomy for persistent PHPT, Tc99m-MIBI-SPECT had a sensitivity of $50 \%$ for glands with a diameter $>1.5 \mathrm{~cm}$ and a sensitivity of $14 \%$ for glands with a diameter $<1.5 \mathrm{~cm}$.

\section{Localization ability in relation to parathyroid pathology}

In patients undergoing initial surgery, $81 \%$ of pathological glands, whether adenomatous or hyperplastic, had a predominance of chief cells and only $19 \%$ had a predominance of oxyphil cells. In patients undergoing reoperative parathyroidectomy for persistent PHPT, 59\% of pathological glands had a predominance of chief cells, $12 \%$ had a predominance of oxyphil cells and the remaining glands had a combination of cell types. In patients who underwent initial surgery for sporadic PHPT, Tc99m-MIBI-SPECT was more sensitive in localizing pathological parathyroid glands with a predominance of oxyphil cells compared to those with a predominance of chief cells (100 vs. 47\%, respectively). This higher sensitivity for pathological parathyroid glands with a predominance of oxyphil cells could not be replicated in patients who underwent reoperative parathyroidectomy for persistent PHPT.

\section{Discussion}

Our findings from the present study underline the limitations of the Tc99m-MIBI-SPECT imaging technique in the accurate preoperative localization of residual pathological parathyroid glands before reoperative parathyroidectomy in patients with persistent hyperparathyroidism. Advances in imaging technology have promoted the development of more focused approaches in the surgical management of primary hyperparathyroidism. The preoperative localization of a pathological parathyroid gland is, however, a prerequisite for a unilateral or minimally invasive surgical approach. Although more than $80 \%$ of patients with sporadic primary hyperparathyroidism will have a single adenoma, it has been estimated that $15-20 \%$ will have a double adenoma or four-gland hyperplasia [32, 33]. Ten percent of individuals may also have supernumerary glands (5-7 glands) [34-36], so that it is not surprising that some of these pathological glands may be missed at initial surgery. It has indeed been reported that $1-7 \%$ of patients with primary hyperparathyroidism will have persistent hyperparathyroidism after initial surgery $[6,37,38]$. When this is the case, it becomes essential to accurately localize the residual hyperactive glands if morbidity from reoperative parathyroidectomy is to be significantly decreased [39].

Limited data suggest that the localizing value of Tc $99 \mathrm{~m}-$ MIBI-SPECT is much lower for the preoperative localization of residual active parathyroid glands before reoperative parathyroidectomy [28-30]. Findings from the present study confirm this notion and emphasize that the ability of Tc99m-MIBI-SPECT to detect and to accurately localize residual hyperactive glands is significantly jeopardized in patients with persistent hyperparathyroidism before reoperative parathyroidectomy compared to its detection and localization ability for pathological parathyroid glands before initial surgery for sporadic PHPT. Several factors may play a role in this decrease in the scan's ability to detect and accurately localize hyperactive parathyroid glands in persistent PHPT. Compared to patients with sporadic PHPT cured after initial surgery, patients with persistent hyperparathyroidism had a lower proportion of adenomas (44 vs. 74\%), a higher proportion of hyperplastic glands (55 vs. 26\%), more ectopically located glands (44 vs. $22 \%$ ), and smaller pathological gland size $(1.21$ vs. $2.03 \mathrm{~cm})$. Published data do indeed suggest a lower predictive value for Tc99m-MIBI-SPECT in cases of parathyroid hyperplasia, ectopic glands, and glands of small size $[10,15,18,19,40,41]$. Patients with persistent HPTH are also at a higher risk from parathyromatosis due to potential spillage during sometimes multiple earlier surgical procedures.

With Tc99m-MIBI imaging, the radiopharmaceutical uptake of pathological parathyroid glands depends not only on perfusion of the hyperactive gland but also on mitochondrial activity of the hyperactive cells. In the parathyroid gland, oxyphil cells have an abundance of mitochondria compared to chief cells. Studies focusing on the causes for negative Tc99m-MIBI-SPECT imaging suggest an influence of the presence of mitochondria-rich cells on outcome. Mihai et al. [18] and Erbil et al. [42] found that Tc99m-MIBI-SPECT scans were more frequently positive than negative in the case of adenomas rich in mitochondria-abundant oxyphil cells. In contrast, Westerdahl and Bergenfelz [19] found no difference in the positive or negative imaging of predominantly oxyphil-rich adenomas.

Parathyroid adenomas are mainly composed of chief cells, and it could be argued that sestamibi may not be taken up by these pathological glands. This is not the case, however, as demonstrated by the frequent finding of both chief cells and oxyphil cells-although in different percentages-in parathyroid adenomas. When both types of cells are found, the net number of mitochondria-rich cells would depend in large part on the size of the pathological gland. The same applies for hyperplastic glands. In keeping with published data, we also observe a clear relationship between gland size and localizing accuracy of Tc99m-MIBI-SPECT. The accuracy of a Tc99m-MIBI-SPECT scan is thus determined mainly by size and amount of oxyphil cells rather than by the 
predominance of a cell type or the adenomatous or hyperplastic nature of the gland.

Our findings from the present study demonstrate that the ability of the widely used Tc99m-MIBI-SPECT to detect and accurately localize pathological parathyroid glands is significantly limited in patients with persistent hyperparathyroidism before reoperative parathyroidectomy. Although the precise cause for this remains unclear, it is likely to be multifactorial, and probably includes disturbances in the local vascular supply caused by previous surgery, as well as differences in gland pathology and size ultimately affecting radiopharmaceutical uptake. It is also conceivable that a previous scan may also have been unable to localize hyperactive parathyroid gland(s) in a specific individual prior to initial surgery. Because most patients in this series did not have preoperative scans before their initial surgery, however, addressing the question of the general ability of Tc99m-MIBI-SPECT to localize a hyperactive parathyroid gland in a specific individual is impossible and represents a limitation of the present study.

Our data hold significant implications in the management of patients with persistent hyperparathyroidism. From the practical point of view, and in keeping with recent guidelines of the European Association of Nuclear Medicine [43], our data suggest that it is always worth performing a Tc99m-MIBI-SPECT scan as a first preoperative localization study in patients with either sporadic or persistent hyperparathyroidism. Should the scan fail to demonstrate increased uptake in neck or mediastinum, no effort should be spared to attempt further localization of residual hyperactive glands prior to surgery to minimize morbidity. For instance, it is possible to use the invasive but more reliable PTH venous sampling technique (our own unpublished observations), followed by a high-resolution 4-dimensional CT scan of the area of interest. Should the scan be able to lateralize the lesion to the right or left side of the neck, it would be reasonable to undertake more limited unilateral neck exploration under the umbrella of intraoperative PTH measurements. Whether the recently advocated use of double-tracer subtraction parathyroid scintigraphy [43] is able to provide better detection and localization accuracy for residual hyperactive parathyroid glands than Tc99m-MIBI-SPECT imaging in the difficult group of patients with persistent hyperparathyroidism remains to be established.

\section{Conflict of interest None.}

Open Access This article is distributed under the terms of the Creative Commons Attribution Noncommercial License which permits any noncommercial use, distribution, and reproduction in any medium, provided the original author(s) and source are credited.

\section{References}

1. Ruda JM, Hollenbeak CS, Stack BC Jr (2005) A systematic review of the diagnosis and treatment of primary hyperparathyroidism from 1995 to 2003. Otolaryngol Head Neck Surg 132: 359-372

2. Simental A, Ferris RL (2008) Reoperative parathyroidectomy. Otolaryngol Clin North Am 41:1269-1274, xii

3. Shen W, Duren M, Morita E et al (1996) Reoperation for persistent or recurrent primary hyperparathyroidism. Arch Surg 131:861-867

4. Hasse C, Sitter H, Brune M et al (2002) Quality of life and patient satisfaction after reoperation for primary hyperparathyroidism: analysis of long-term results. World J Surg 26:1029-1036

5. Gough I (2006) Reoperative parathyroid surgery: the importance of ectopic location and multigland disease. Aust N Z J Surg 76:1048-1050

6. Witteveen JE, Kievit J, Morreau H et al (2010) No recurrence of sporadic primary hyperparathyroidism when cure is established 6 months after parathyroidectomy. Eur J Endocrinol 162: 399-406

7. Westerdahl J, Bergenfelz A (2007) Unilateral versus bilateral neck exploration for primary hyperparathyroidism: five-year follow-up of a randomized controlled trial. Ann Surg 246: 976-980

8. Carty SE (2004) Prevention and management of complications in parathyroid surgery. Otolaryngol Clin North Am 37:897-907, xi

9. Brenner ME, Jacene HA (2008) Recurrent or residual hyperparathyroidism and thyroid cancer effectively evaluated with scintigraphy. Otolaryngol Clin North Am 41:1117-1119

10. Carneiro-Pla DM, Solorzano CC, Irvin GL III (2006) Consequences of targeted parathyroidectomy guided by localization studies without intraoperative parathyroid hormone monitoring. J Am Coll Surg 202:715-722

11. Chiu B, Sturgeon C, Angelos P (2006) What is the link between nonlocalizing sestamibi scans, multigland disease, and persistent hypercalcemia? A study of 401 consecutive patients undergoing parathyroidectomy. Surgery 140:418-422

12. Lo CY, Lang BH, Chan WF et al (2007) A prospective evaluation of preoperative localization by technetium- $99 \mathrm{~m}$ sestamibi scintigraphy and ultrasonography in primary hyperparathyroidism. Am J Surg 193:155-159

13. Palmer RM, Lokey JS (2006) Is minimally invasive parathyroidectomy reasonable in the nonuniversity setting? Am J Surg 192:865-868

14. Calva-Cerqueira D, Smith BJ, Hostetler ML et al (2007) Minimally invasive parathyroidectomy and preoperative MIBI scans: correlation of gland weight and preoperative PTH. J Am Coll Surg 205:S38-S44

15. Jones JM, Russell CF, Ferguson WR et al (2001) Pre-operative sestamibi-technetium subtraction scintigraphy in primary hyperparathyroidism: experience with 156 consecutive patients. Clin Radiol 56:556-559

16. Moka D, Voth E, Dietlein M et al (2000) Technetium 99m-MIBISPECT: a highly sensitive diagnostic tool for localization of parathyroid adenomas. Surgery 128:29-35

17. Bergenfelz A, Lindblom P, Tibblin S et al (2002) Unilateral versus bilateral neck exploration for primary hyperparathyroidism: a prospective randomized controlled trial. Ann Surg 236: 543-551

18. Mihai R, Gleeson F, Buley ID et al (2006) Negative imaging studies for primary hyperparathyroidism are unavoidable: correlation of sestamibi and high-resolution ultrasound scanning with histological analysis in 150 patients. World J Surg 30:697-704 
19. Westerdahl J, Bergenfelz A (2004) Sestamibi scan-directed parathyroid surgery: potentially high failure rate without measurement of intraoperative parathyroid hormone. World J Surg 28:1132-1138

20. Sugg SL, Krzywda EA, Demeure MJ et al (2004) Detection of multiple gland primary hyperparathyroidism in the era of minimally invasive parathyroidectomy. Surgery 136:1303-1309

21. Arbab AS, Koizumi K, Toyama K et al (1996) Uptake of technetium-99m-tetrofosmin, technetium-99m-MIBI and thallium201 in tumor cell lines. J Nucl Med 37:1551-1556

22. Adams BK, Fataar A, Nizami MA (1996) Technetium-99msestamibi uptake in myeloma. J Nucl Med 37:1001-1002

23. Feingold DL, Alexander HR, Chen CC et al (2000) Ultrasound and sestamibi scan as the only preoperative imaging tests in reoperation for parathyroid adenomas. Surgery 128:1103-1109

24. Thompson GB, Grant CS, Perrier ND et al (1999) Reoperative parathyroid surgery in the era of sestamibi scanning and intraoperative parathyroid hormone monitoring. Arch Surg 134:699-704

25. Hessman O, Stalberg P, Sundin A et al (2008) High success rate of parathyroid reoperation may be achieved with improved localization diagnosis. World J Surg 32:774-781

26. Numerow LM, Morita ET, Clark OH et al (1995) Persistent/ recurrent hyperparathyroidism: a comparison of sestamibi scintigraphy, MRI, and ultrasonography. J Magn Reson Imaging 5:702-708

27. Majors JD, Burke GJ, Mansberger AR Jr et al (1995) Technetium Tc99m sestamibi scan for localizing abnormal parathyroid glands after previous neck operations: preliminary experience in reoperative cases. South Med J 88:327-330

28. Rotstein L, Irish J, Gullane P et al (1998) Reoperative parathyroidectomy in the era of localization technology. Head Neck 20:535-539

29. Chen CC, Skarulis MC, Fraker DL et al (1995) Technetium-99msestamibi imaging before reoperation for primary hyperparathyroidism. J Nucl Med 36:2186-2191

30. Fayet P, Hoeffel C, Fulla Y et al (1997) Technetium-99m sestamibi scintigraphy, magnetic resonance imaging and venous blood sampling in persistent and recurrent hyperparathyroidism. Br J Radiol 70:459-464

31. DeLellis RA (2006) World health organisation classification of tumours. Pathology and genetics of tumours of endocrine organs. ARC Press, Lyon, France
32. Hamdy NA (2007) A patient with persistent primary hyperparathyroidism due to a second ectopic adenoma. Nat Clin Pract Endocrinol Metab 3:311-315

33. Udelsman R, Pasieka JL, Sturgeon C et al (2009) Surgery for asymptomatic primary hyperparathyroidism: proceedings of the third international workshop. J Clin Endocrinol Metab 94: 366-372

34. Grimelius L, Akerstrom G, Johansson H et al (1981) Anatomy and histopathology of human parathyroid glands. Pathol Annu $16: 1-24$

35. Akerstrom G, Rudberg C, Grimelius L et al (1992) Causes of failed primary exploration and technical aspects of re-operation in primary hyperparathyroidism. World J Surg 16:562-568

36. Wang C (1976) The anatomic basis of parathyroid surgery. Ann Surg 183:271-275

37. Yen TW, Wang TS, Doffek KM et al (2008) Reoperative parathyroidectomy: an algorithm for imaging and monitoring of intraoperative parathyroid hormone levels that results in a successful focused approach. Surgery 144:611-619

38. Richards ML, Thompson GB, Farley DR et al (2008) Reoperative parathyroidectomy in 228 patients during the era of minimalaccess surgery and intraoperative parathyroid hormone monitoring. Am J Surg 196:937-942

39. Carty SE, Norton JA (1991) Management of patients with persistent or recurrent primary hyperparathyroidism. World J Surg 15:716-723

40. Lumachi F, Zucchetta P, Marzola MC et al (2000) Advantages of combined technetium-99m-sestamibi scintigraphy and high-resolution ultrasonography in parathyroid localization: comparative study in 91 patients with primary hyperparathyroidism. Eur J Endocrinol 143:755-760

41. Allendorf J, Kim L, Chabot J et al (2003) The impact of sestamibi scanning on the outcome of parathyroid surgery. J Clin Endocrinol Metab 88:3015-3018

42. Erbil Y, Kapran Y, Issever H et al (2008) The positive effect of adenoma weight and oxyphil cell content on preoperative localization with $99 \mathrm{mTc}$-sestamibi scanning for primary hyperparathyroidism. Am J Surg 195:34-39

43. Hindie E, Ugur O, Fuster D et al (2009) 2009 EANM parathyroid guidelines. Eur J Nucl Med Mol Imaging 36:1201-1216 